\title{
Are quantitative histopathologic criteria capable of differentiating psoriasis from chronic dermatitis?
}

This article was published in the following Dove Press journal:

Clinical, Cosmetic and Investigational Dermatology

\author{
Hamid Reza Ghasemi \\ Basir ${ }^{1,2}$ \\ Pedram Alirezaei ${ }^{2}$ \\ Zahra Hamian ${ }^{3}$ \\ Elham Khanlarzadeh ${ }^{4}$ \\ 'Department of Pathology, School \\ of Medicine, Hamadan University \\ of Medical Sciences, Hamadan, Iran; \\ ${ }^{2}$ Psoriasis Research Center, Hamadan \\ University of Medical Sciences, \\ Hamadan, Iran; ${ }^{3}$ School of Medicine, \\ Hamadan University of Medical \\ Sciences, Hamadan, Iran; ${ }^{4}$ Department \\ of Community Medicine, School of \\ Medicine, Hamadan University of \\ Medical Sciences, Hamadan, Iran
}

Background: There are overlapping features in histopathologic characteristics of psoriasis and chronic dermatitis, which sometimes make the correct diagnosis difficult.

Objective: The aim of this study was to compare the histopathologic diagnostic features of psoriasis with chronic dermatitis quantitatively.

Patients and methods: In this study, 30 patients with psoriasis and 30 patients with chronic dermatitis were included. Diagnosis of psoriasis or chronic dermatitis was based on clinicopathologic correlation. Photos of histopathologic slides were provided by LABOMED Digital Camera and LABOMED Microscope. Width and length of rete ridges, minimal thickness of suprapapillary plates, thickness of epidermis, thickness of hyperkeratotic layer, and minimal thickness of granular layer were determined using Pixel Pro software on micrometer scale.

Results: Suprapapillary plates and granular layer were significantly thicker in patients with chronic dermatitis compared with patients with psoriasis. The rete ridges were longer and wider, the epidermis was thicker, and the parakeratotic layer was also thicker in patients with psoriasis compared with patients with chronic dermatitis, and the difference between these corresponding parameters in the two groups was statistically significant except for thickness of parakeratotic layer. Moreover, the mean ratio of minimal suprapapillary plate's thickness to epidermal thickness and the mean ratio of minimal granular layer's thickness to parakeratotic layer's thickness were significantly higher in patients with chronic dermatitis compared with those with psoriasis. Conclusion: Despite overlapping histopathologic features in psoriasis and chronic dermatitis, quantitative histopathologic criteria might be valuable for differentiation of these two conditions. Ratios of minimal suprapapillary plate's thickness to epidermal thickness and minimal granular layer's thickness to parakeratotic layer's thickness can be particularly helpful in this regard.

Keywords: psoriasis, chronic dermatitis, histopathology, quantitative

\section{Introduction}

Psoriasis is an autoimmune chronic skin disease that may sometimes significantly impair quality of life. ${ }^{1-3}$ Overall prevalence of psoriasis in general population is different in different parts of the world, and some recent studies claim that its prevalence is increasing. ${ }^{4}$ In western world, an estimated $2 \%-4 \%$ of general population are affected by the disease. ${ }^{5}$ Moreover, psoriasis has been associated with several comorbidities (including cardiovascular, metabolic, and rheumatologic disorders), and these comorbidities are more prevalent in patients with more severe skin disease ${ }^{6-19}$ Most researchers believe that psoriasis has multifactorial etiology, and both genetic and environmental factors contribute to its etiopathogenesis..$^{20}$ The most common clinical form of psoriasis is psoriasis vulgaris, characterized by symmetrically distributed
Correspondence: Pedram Alirezaei Psoriasis Research Center, Farshchian Hospital, Mirzadeh Eshghi Street, Hamadan, Iran

$\mathrm{Tel} / \mathrm{Fax}+988138272154$

Email prc@umsha.ac.ir 
erythematous plaques covered with scale. ${ }^{21}$ In microscopic evaluation, a significant increase in epidermal thickness (acanthosis), downward extension of rete ridges, diminished granular layer, parakeratosis, thinning of suprapapillary plates, dilatation and tortuosity of capillaries in papillary dermis, Munro's neutrophilic microabscesses and spongiform pustules of Kogoj constitute the histopathologic characteristics of psoriatic lesion. However, during different stages of evolution of a psoriatic plaque, only some of these features might be present. Munro's neutrophilic microabscesses and spongiform pustules of Kogoj are considered to be diagnostic for psoriasis, but sometimes they might be absent. ${ }^{22}$

Chronic dermatitis is clinically characterized by itching, scratching, ulceration, and lichenification of skin. ${ }^{23}$ Its histopathologic changes include acanthosis, hyperkeratosis, spongiosis, lymphocytic exocytosis, superficial dermal fibrosis, and perivascular lymphocytic infiltration. ${ }^{24}$

Although some findings such as Munro's microabscesses and thinning of the granular layer are more common in psoriatic lesion, differentiating psoriasis from chronic dermatitis is generally difficult because many of their histologic features are identical. ${ }^{22}$ Some studies had previously tried to clarify the histopathologic differences between psoriasis and chronic dermatitis, but their findings are not completely consistent. $^{25,26}$ Furthermore, they had not used quantitative histopathologic methods to distinguish between these two diseases.

The aim of our study was to compare the histopathologic diagnostic features of psoriasis with chronic dermatitis quantitatively, in order to make the differentiation easier.

\section{Patients and methods}

This cross-sectional study was conducted in Hamadan, west of Iran. The ethics committee of Hamadan University of Medical Sciences approved the study protocol (ethics committee code: IR.UMSHA.REC.1395.271), and written informed consent was obtained from all participants. Thirty patients with clinical diagnosis of psoriasis and 30 patients with clinical diagnosis of chronic dermatitis, whose biopsies also confirmed the clinical diagnosis, were included in the study. The definite diagnosis was based on clinicopathologic correlation (only individuals with typical clinical features along with typical histopathologic findings of psoriasis were included in the psoriasis group and vice versa). Demographic characteristics such as age and gender, as well as clinical information were recorded. Photos of histologic slides were obtained using LABOMED microscope and digital camera (LaboAmerica, Fremont, CA, USA) at two magnifications $(\times 100$ and $\times 400)$. A computer was connected to the camera, and Pixel Pro software (Labomed, Los Angeles, CA, USA) was installed on the computer for imaging and microscopic measurements. Width and length of rete ridges (at the longest rete ridge), minimal thickness of suprapapillary plates (defined as the shortest distance between the top of dermal papilla and cornified layer), thickness of epidermis (at the thickest part), thickness of hyperkeratotic layer (the thickest part of parakeratotic or orthokeratotic hyperkeratosis), and minimal thickness of granular layer (the thinnest portion of granular layer in the parakeratotic area) were determined using Pixel Pro software on the micrometer scale by a pathologist (Figure 1A and B).

In descriptive statistics section, mean and SD were used to report quantitative variables with normal distribution, while median was used for the remaining quantitative variables. Qualitative variables were also described using ratio and percentage. To compare the quantitative variables in two groups, independent samples $t$-test was used if the distribution was normal. For those variables without normal distribution, nonparametric tests (including Mann-Whitney $U$-test) were used. To compare the qualitative-nominal variables in the two groups (such as gender and anatomic

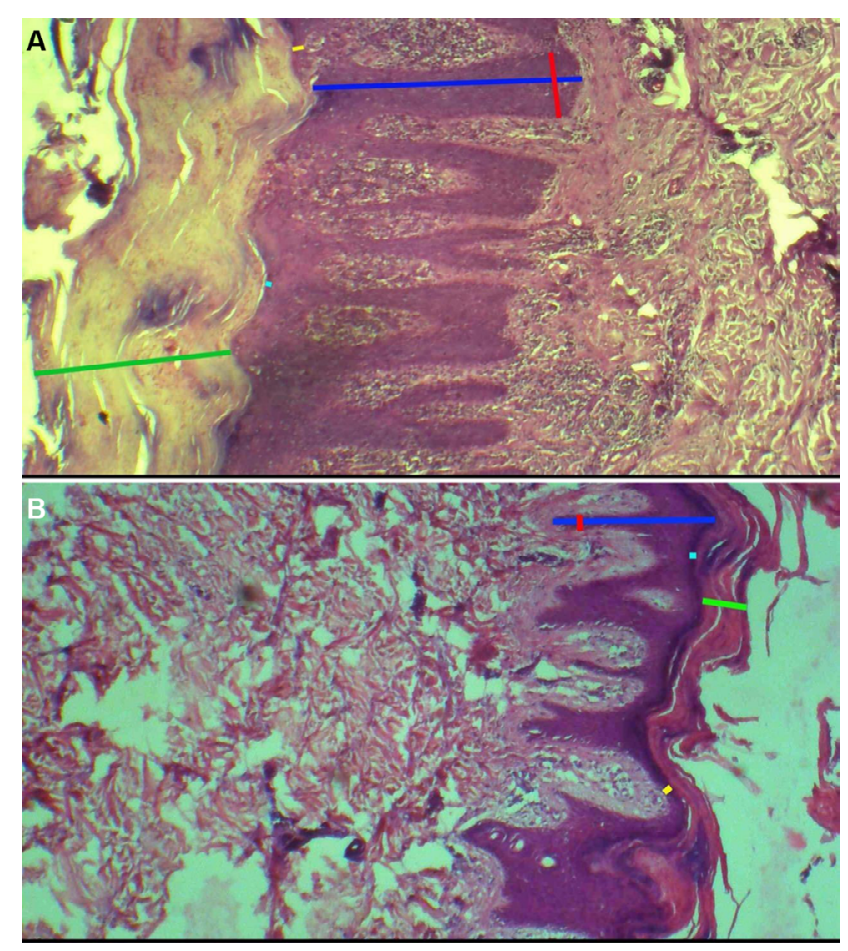

Figure I (A) Histopathology of psoriasis $(\mathrm{H} \& \mathrm{E} \times 100)$. (B) Histopathology of chronic dermatitis $(\mathrm{H} \& \mathrm{E} \times 100)$.

Notes: Width of the longest rete ridge; minimal thickness of granular layer; length of the longest rete ridge; thickest part of parakeratosis; minimal thickness of suprapapillary plate. 
location), chi-square test or Fisher's exact test was used. All the statistical analyses were carried out using SPSS 16.0 for Windows (SPSS Inc., Chicago, IL, USA) and $p$-values $<0.05$ were considered significant.

\section{Results}

Thirty patients with psoriasis and 30 patients with chronic dermatitis were included in this study. Twenty-one patients with dermatitis were female (70\%), while in the psoriasis group, 20 patients $(66.7 \%)$ were male. The mean age of patients in the dermatitis group was not significantly different from the mean age of patients in the psoriasis group (41.86 \pm 17.44 versus $38.2 \pm 14.03$ years, respectively). The most common anatomical location was upper limb in the dermatitis group $(33.3 \%)$, while in the psoriasis group, it was lower extremity (36.7\%).

The mean length of rete ridge in patients with psoriasis was higher than those with chronic dermatitis (4479.6 versus $2369.4 \mu \mathrm{m}$, respectively [ $p=0.001])$. The mean width of rete ridge in patients with dermatitis was lower than those with psoriasis (580.4 versus $1053.9 \mu \mathrm{m}$, respectively [ $p=0.001]$ ).

The mean thickness of suprapapillary plate in patients with psoriasis was lower than that in patients with chronic dermatitis (498.2 versus $621.6 \mu \mathrm{m}$, respectively [ $p=0.022]$ ). Moreover, the average thickness of epidermis was higher in patients with psoriasis than that in patients with chronic dermatitis (5047.2 versus $2487.6 \mu \mathrm{m}$, respectively [ $p=0.001]$ ).

The average thickness of granular layer in patients with chronic dermatitis was $133.9 \mu \mathrm{m}$, while in patients with psoriasis it was $41.7 \mu \mathrm{m}(p=0.001)$. The mean thickness of parakeratotic layer in patients with chronic dermatitis was $1130.8 \mu \mathrm{m}$ and in patients with psoriasis, it was $1228.6 \mu \mathrm{m}$, but this difference was not statistically significant ( $p=0.094$; Table 1).

The mean width to length ratio of rete ridge in patients with chronic dermatitis was lower than that in patients with psoriasis, but this difference was not significant $(p=0.964)$. The mean ratio of suprapapillary plate's thickness to epidermal thickness and the mean ratio of granular layer's thickness to parakeratotic layer's thickness were higher in patients with chronic dermatitis than in those with psoriasis ( $p=0.001$ for both; Table 2).

In receiver operating characteristic curve analysis, a ratio of 0.06 for granular layer's thickness to parakeratotic layer's thickness was the best cutoff point (sensitivity of $90 \%$ and specificity of $80 \%$ ), thus the ratios $\leq 0.06$ were in favor of the diagnosis of psoriasis (Figure 2). Moreover, a ratio of 0.13 for suprapapillary plate's thickness to epidermal thickness was the best cutoff point (sensitivity of $80 \%$ and specificity of $90 \%$ ), thus the ratios $\leq 0.13$ were in favor of the diagnosis of psoriasis (Figure 3).

\section{Discussion}

Psoriasis is a chronic inflammatory skin disease with characteristic clinicopathologic findings, but its differentiation from chronic dermatitis may sometimes become difficult.

We showed that the thickness of suprapapillary plate and granular layer was greater in patients with chronic dermatitis

Table I Comparison of quantitative histopathologic parameters between chronic dermatitis and psoriasis

\begin{tabular}{|c|c|c|c|c|c|c|c|c|c|}
\hline \multirow[t]{2}{*}{ Variables } & \multicolumn{4}{|c|}{ Psoriasis } & \multicolumn{4}{|c|}{ Dermatitis } & \multirow{2}{*}{$\begin{array}{l}\text { P-value } \\
\text { (Mann- } \\
\text { Whitney) }\end{array}$} \\
\hline & Mean & SD & Min & Max & Mean & SD & Min & Max & \\
\hline Rete ridge length $(\mu \mathrm{m})$ & 4479.6 & 1438.3 & 2388.9 & 8177.5 & 2369.4 & 849.8 & 1318.4 & 4810.5 & 0.001 \\
\hline Rete ridge width $(\mu \mathrm{m})$ & 1053.9 & 369.3 & 355.9 & 1800.6 & 580.4 & 279.1 & 264.1 & 1160.7 & 0.001 \\
\hline Thickness of suprapapillary plate $(\mu \mathrm{m})$ & 498.2 & 218.5 & 67.5 & 4371.6 & 621.6 & 739.1 & 248.7 & 4431.8 & 0.022 \\
\hline Thickness of epidermis $(\mu \mathrm{m})$ & 5047.2 & 1478.6 & 2763.2 & 8625.3 & 2487.6 & 883.1 & 1318.4 & 4810.5 & 0.001 \\
\hline Thickness of granular layer $(\mu \mathrm{m})$ & 41.7 & 31.9 & 0 & 107.9 & 133.9 & 55.2 & 48.4 & 294.4 & 0.001 \\
\hline Thickness of parakeratotic layer $(\mu \mathrm{m})$ & 1228.6 & 1014.5 & 400.5 & 5764.3 & 1130.8 & 1181.1 & 228.4 & 5377.8 & 0.094 \\
\hline
\end{tabular}

Table 2 Comparison of quantitative histopathologic parameter ratios between chronic dermatitis and psoriasis

\begin{tabular}{|c|c|c|c|c|c|c|c|c|c|}
\hline \multirow[t]{2}{*}{ Ratios } & \multicolumn{4}{|c|}{ Psoriasis } & \multicolumn{4}{|c|}{ Dermatitis } & \multirow{2}{*}{$\begin{array}{l}\text { P-value } \\
\text { (Mann- } \\
\text { Whitney) }\end{array}$} \\
\hline & Mean & SD & Min & $\operatorname{Max}$ & Mean & SD & Min & $\operatorname{Max}$ & \\
\hline Width to length of rete ridge & 0.256 & 0.116 & 0.063 & 0.533 & 0.251 & 0.109 & 0.109 & 0.564 & 0.964 \\
\hline Suprapapillary plate's thickness to epidermal thickness & 0.120 & 0.226 & 0.010 & 1.29 & 0.278 & 0.377 & 0.082 & 2.23 & 0.001 \\
\hline Granular layer's thickness to parakeratotic layer's thickness & 0.045 & 0.042 & 0 & 0.133 & 0.182 & 0.113 & 0.050 & 0.500 & 0.001 \\
\hline
\end{tabular}




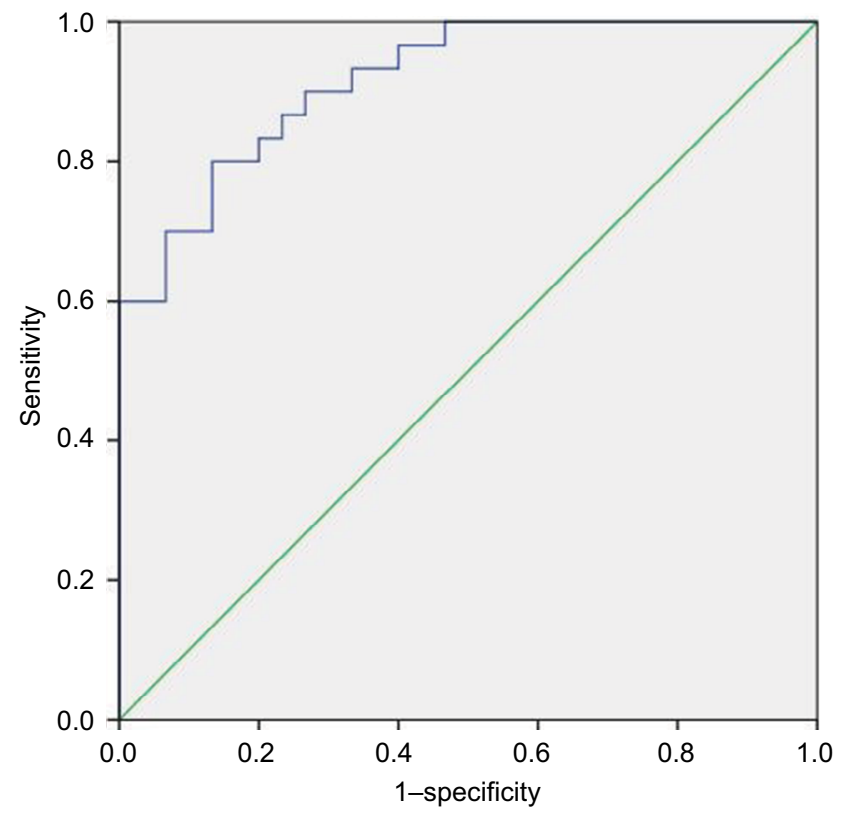

Figure 2 ROC curve for granular layer's thickness to parakeratotic layer's thickness ratio.

Abbreviation: ROC, receiver operating characteristic.

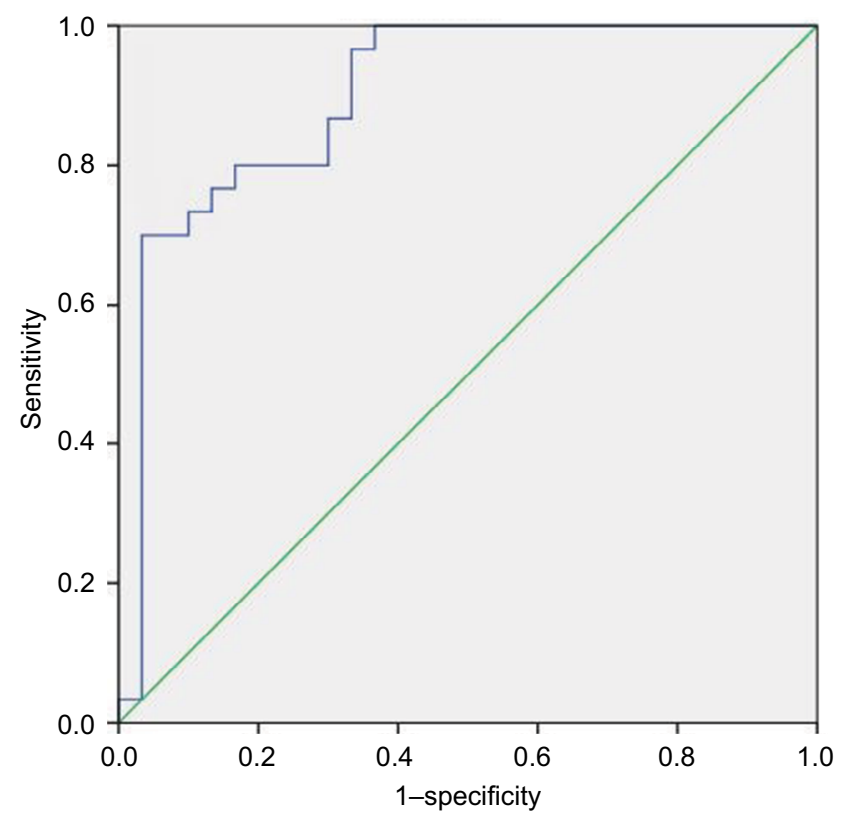

Figure 3 ROC curve for suprapapillary plate's thickness to epidermal thickness ratio.

Abbreviation: ROC, receiver operating characteristic.

compared with patients with psoriasis $(p=0.022, p=0.001$, respectively). We also demonstrated that the length and the width of rete ridge, the epidermal thickness, and the thickness of parakeratosis were greater in patients with psoriasis compared with those with chronic dermatitis, and the differences were significant except for the thickness of parakeratosis $(p=0.001$, $p=0.001, p=0.001, p=0.094$, respectively). Kamyab-Hesari et al have shown that in addition to Munro's microabscesses and tortuosity of capillaries in the papillary dermis, hypogranulosis, suprapapillary thinning, and confluent parakeratosis were significantly in favor of the diagnosis of psoriasis. ${ }^{25}$ Although their findings have not been quantitatively reported, they are consistent with ours. In another study conducted by Aydin et al $41.2 \%$ of patients with psoriasis had no granular layer compared to $36 \%$ of patients with chronic dermatitis. ${ }^{26}$ Our quantitative findings are also consistent with their results. These findings point toward the importance of the loss of granular layer in differentiating psoriasis from dermatitis.

In our study, the thickness of parakeratotic layer was slightly greater in patients with psoriasis compared with those with chronic dermatitis, but the difference was not statistically significant. It should be noted that this parameter may be affected by excoriation in both conditions, and therefore it is not a reliable distinguishing factor.

Based on our findings, the suprapapillary plates were thinner in patients with psoriasis compared with those with chronic dermatitis $(p=0.022)$. In the study conducted by Aydin et al, the suprapapillary thinning was also found to be more common in patients with psoriasis compared with those with dermatitis, but the difference was not significant. The absence of a significant difference in their study may be due to lack of quantitative measurements.

The findings of our study are also consistent with the study conducted by Mehta et al, in which suprapapillary thinning and the absence of granular layer were shown to be significantly more common in patients with psoriasis. ${ }^{27}$

Given the histopathologic nature of psoriasis and chronic dermatitis, these findings are not unexpected. However, it should be kept in mind that the presence or absence of all these typical histologic features depends on the age of the lesion, the site of sampling, and the presence or absence of excoriation. The site of sampling is particularly important, because it can affect considerably the interpretation of epidermal thickness, length of rete ridges, granular layer's thickness, and cornified layer's thickness. In order to remove the confounding effects of this variable, we decided to use three ratios: width to length of rete ridge, thickness of suprapapillary plate to epidermal thickness, and granular layer's thickness to parakeratotic layer's thickness.

Although the mean ratio of width to length of rete ridge was lower in patients with chronic dermatitis compared with those with psoriasis, this difference was not statistically significant ( $p=0.964)$. We hypothesized that this is due to simultaneous increase in length and width of rete ridges in psoriasis. 
The mean ratio of suprapapillary plate's thickness to epidermal thickness and the mean ratio of granular layer's thickness to parakeratotic layer's thickness were higher in patients with chronic dermatitis compared with those with psoriasis ( $p=0.001$ for both). This may be due to a significant difference in the thickness of suprapapillary plate and granular layer between the two groups.

\section{Limitation}

A major limitation of this study is that we examined typical cases of psoriasis and chronic dermatitis, while histopathology is mostly needed in borderline cases. However, our study is merely a starting point, and we had to begin with typical cases, but future studies should definitely concentrate on borderline ones.

\section{Conclusion}

The major differential diagnosis of psoriasis, both clinically and histopathologically, is chronic dermatitis. Despite the overlapping histopathologic features, some findings (such as diminished granular layer, parakeratosis, suprapapillary thinning, and Munro's microabscesses) are in favor of the diagnosis of psoriasis. Unfortunately, all these parameters may be affected by the severity and duration of disease, secondary changes due to excoriation, and anatomical location of biopsy. We showed that by using quantitative parameters, diagnostic accuracy may be increased. Specifically, using the cutoff point of 0.06 for the ratio of granular layer's thickness to parakeratotic layer's thickness and 0.13 for the ratio of suprapapillary plate's thickness to epidermal thickness may help to differentiate psoriasis from chronic dermatitis. In other words, ratios $\leq 0.06$ for granular layer's thickness to parakeratotic layer's thickness and $\leq 0.13$ for suprapapillary plate's thickness to epidermal thickness are in favor of the diagnosis of psoriasis.

\section{Acknowledgments}

This study was conducted based on the approved MD thesis of Zahra Hamian (thesis no.: 9506023239), and we would like to thank the vice-chancellor for research and technology at Hamadan University of Medical Sciences for thesis approval.

\section{Disclosure}

The authors report no conflicts of interest in this work.

\section{References}

1. Lopes N, Pietri G, Howe T, et al. PSS38 - Psoriasis (PSO) patients' profile and impact of the disease on patients' quality of life and work productivity in a real-life setting in Brazil. Value Health.2017;20(9):A807.
2. Gupta MA, Gupta AK. Quality of life of psoriasis patients. J Eur Acad Dermatol Venereol. 2000;14:241-242.

3. Kaufman BP, Alexis AF. Psoriasis in skin of color: insights into the epidemiology, clinical presentation, genetics, quality-of-life impact, and Treatment of Psoriasis in Non-White Racial/Ethnic Groups. Am J Clin Dermatol. Epub 2017 Dec 5.

4. Danielsen K, Olsen AO, Wilsgaard T, Furberg AS. Is the prevalence of psoriasis increasing? A 30-year follow-up of a population-based cohort. Br J Dermatol. 2013;168(6):1303-1310.

5. Parisi R, Symmons DP, Griffiths CE, Ashcroft DM; Identification and Management of Psoriasis and Associated ComorbidiTy (IMPACT) project team. Global epidemiology of psoriasis: a systematic review of incidence and prevalence. J Invest Dermatol. 2013;133(2): 377-385.

6. Yeung H, Takeshita J, Mehta NN, et al. Psoriasis severity and the prevalence of major medical comorbidity: a population-based study. JAMA Dermatol. 2013;149(10):1173-1179.

7. Dattilo G, Borgia F, Guarneri C, et al. Cardiovascular risk in psoriasis: current state of the art. Curr Vasc Pharmacol. Epub 2017 Nov 16.

8. Dattilo G, Imbalzano E, Casale M, et al. Psoriasis and cardiovascular risk: correlation between psoriasis and cardiovascular functional indices. Angiology. 2018;69(1):31-37.

9. Egeberg A, Skov L, Joshi AA, et al. The relationship between duration of psoriasis, vascular inflammation, and cardiovascular events. $J \mathrm{Am}$ Acad Dermatol. 2017;77(4):650-656.e653.

10. Furue M, Tsuji G, Chiba T, Kadono T. Cardiovascular and metabolic diseases comorbid with psoriasis: beyond the skin. Intern Med. 2017;56(13): $1613-1619$.

11. Hu SC, Lan CE. Psoriasis and cardiovascular comorbidities: focusing on severe vascular events, cardiovascular risk factors and implications for treatment. Int J Mol Sci. 2017;18(10):E2211.

12. Gisondi P, Fostini AC, Fossa I, Girolomoni G, Targher G. Psoriasis and the metabolic syndrome. Clin Dermatol. 2018;36(1):21-28.

13. Gui XY, Yu XL, Jin HZ, Zuo YG, Wu C. Prevalence of metabolic syndrome in Chinese psoriasis patients: a hospital-based cross-sectional study. J Diabetes Investig. 2018;9(1):39-43.

14. Rodriguez-Zuniga MJM, Garcia-Perdomo HA. Systematic review and meta-analysis of the association between psoriasis and metabolic syndrome. J Am Acad Dermatol. 2017;77(4):657-666.e658.

15. Salunke AS, Nagargoje MV, Belgaumkar VA, Tolat SN, Chavan RB. Association of metabolic syndrome in chronic plaque psoriasis patients and their correlation with disease severity, duration and age: a Case Control Study from Western Maharashtra. J Clin Diagn Res. 2017;11(8):WC06-WC10.

16. Singh S, Young P, Armstrong AW. An update on psoriasis and metabolic syndrome: a meta-analysis of observational studies. PLoS One. 2017;12(7):e0181039.

17. Zuo YG, Wu C, Descalzo MA. Psoriasis, metabolic syndrome, and systematic reviews. J Diabetes Investig. 2017;108:323.

18. Carubbi F, Chimenti MS, Blasetti G, et al. Association of psoriasis and/ or psoriatic arthritis with autoimmune diseases: the experience of two Italian integrated Dermatology/Rheumatology outpatient clinics. J Eur Acad Dermatol Venereol. 2015;29(11):2160-2168.

19. Mody E, Husni ME, Schur P, Qureshi AA. Multidisciplinary evaluation of patients with psoriasis presenting with musculoskeletal pain: a dermatology: rheumatology clinic experience. Br J Dermatol. 2007;157(5): 1050-1051.

20. Di Meglio P, Villanova F, Nestle FO. Psoriasis. Cold Spring Harb Perspect Med. 2014;4(8):A015354.

21. Sarac G, Koca TT, Baglan T. A brief summary of clinical types of psoriasis. North Clin Istanb. 2016;3(1):79-82.

22. De Rosa G, Mignogna C. The histopathology of psoriasis. Reumatismo. 2007;59 (Suppl 1):46-48.

23. Miyoshi H, Kanekura T, Aoki T, Kanzaki T. Beneficial effects of tissue inhibitor of metalloproteinases-2 (TIMP-2) on chronic dermatitis. J Dermatol. 2005;32(5):346-353. 
24. Le TK, Schalkwijk J, van de Kerkhof PC, van Haelst U, van der Valk PG. A histological and immunohistochemical study on chronic irritant contact dermatitis. Am J Contact Dermat. 1998;9(1):23-28.

25. Kamyab-Hesari K, Safaei-Naraghi Z, Ghanadan A, Nikoo A, Sabaghi M. Palmoplantar psoriasis versus eczema: major histopathologic clues for diagnosis. Iran J Pathol. 2014;9(4):251-256.
26. Aydin O, Engin B, Oguz O, Ilvan S, Demirkesen C. Non-pustular palmoplantar psoriasis: is histologic differentiation from eczematous dermatitis possible? J Cutan Pathol. 2008;35(2):169-173.

27. Mehta S, Singal A, Singh N, Bhattacharya SN. A study of clinicohistopathological correlation in patients of psoriasis and psoriasiform dermatitis. Indian J Dermatol Venereol Leprol. 2009;75(1):100.

\section{Publish your work in this journal}

Clinical, Cosmetic and Investigational Dermatology is an international, peer-reviewed, open access, online journal that focuses on the latest clinical and experimental research in all aspects of skin disease and cosmetic interventions. This journal is included on PubMed. The manuscript management system is completely online and includes a very quick and fair peer-review system, which is all easy to use. Visit http://www.dovepress.com/testimonials.php to read real quotes from published authors 